\title{
ADAPTIVE SUBSPACE DETECTORS FOR OFF-GRID MISMATCHED TARGETS
}

\author{
Jonathan Bosse ${ }^{1}$, Olivier Rabaste ${ }^{1}$ and Jean-Philippe Ovarlez ${ }^{1,2}$ \\ ${ }^{1}$ :DEMR, ONERA, Université Paris Saclay, F-91123 Palaiseau - France \\ ${ }^{2}$ :SONDRA, Centrale-Supélec, Université Paris-Saclay, Gif-Sur-yvette - France
}

\begin{abstract}
In classical detection framework, the parameter space is usually discretized, so that in reality received parameter dependent signals are never perfectly aligned with the signal model under test: it leads to the off-grid signal mismatch. In a Gaussian adaptive context (i.e. the noise covariance is unknown), Kelly GLRT and AMF detectors are well established techniques that can suffer severe performance degradation in presence of this kind of mismatch. We propose here to use adaptive subspace detectors to solve this issue, a suitable subspace (that coincides with the Discrete Prolate Spheroidal Sequences basis when the signal model is that of sinusoids in noise) is proposed that offers robust performance. The interest lies in the fact that such detectors are really easy to implement and we are able to derive their analytic performance.
\end{abstract}

Index Terms - Off-grid mismatch, adaptive radar detection, Kelly GLRT, AMF, DPSS

\section{INTRODUCTION}

Classically, in a lot of signal processing problems, the signal parameters are assumed discrete whereas the observed scene is often continuous by nature. This may lead to a mismatch between the received parameter dependent signal and the presumed signal model, known as "off-grid" mismatch, which is for example a topic of interest in sparse signal recovery [1].

Signal mismatch has been present in a variety of studies in detection problems. Often, the considered mismatch, designed to reflect uncertainties on the received signals, have very general formulation (e.g. $[2,3])$. On the contrary, the off-grid mismatch is very structured. As a consequence lots of these studies are not specifically designed for the problem under consideration here and might not perform very well. Also, the increased robustness is often obtained at the price of increased computational complexity. To the best of our knowledge, the issue of off-grid target detection has not been the subject of numerous studies in the literature [4].

In this paper we focus on adaptive detection in Gaussian environment in presence of signal mismatch. In absence of mismatch, Kelly's GLRT [5] and Adaptive Matched Filter (AMF) [6] are very widespread approaches. It was already observed that Kelly's test is not very robust to signal mis- match angle [6]. As we will see, in case of off-grid targets the performance degradation can be severe.

Study [3] is, to our knowledge, the closest state of the art. The proposed detector assumes that the signal mismatch angle is bounded (the signals must belong to a defined cone). As a drawback, an optimization has to be performed to compute the measurement projection on the cone, and this should be performed for each new measurement to be tested. Also, analytic probability of detection and false alarm are not known. It is also worth mentioning that the projection to the cone imposes a lower bound on the false alarm probability (since every realization inside the cone is declared as a detection): depending on the problem, this might not be compatible for certain applications (such as radar) where the required false alarm probability can be very low (of the order of $10^{-6}$ ).

We extend the approach in [4] to the case of adaptive detection. In this paper, the idea is to use subspace detector that can be more robust to the off-grid mismatch. We discuss the choice of a good basis and the choice of its dimension. We show that for the widespread sinusoids-in-noise model, the basis is directly deduced from the Discrete Prolate Spheroidal Sequences (DPSS) vectors [7]. The strong advantages of this subspace approach is that associated detectors are easy to implement and it is possible to derive their analytic performances.

The paper is organized as follows : the signal model is presented and the problem is formulated in section 2. Section 3 deals with the adaptive subspace detectors and we provide their performances under off-grid signal mismatch. Section 4 deals with the relevant choice of the subspace and finally section 5 concludes with numerical experiments where we show the benefit of the proposed detectors.

\section{SIGNAL MODEL AND PROBLEM FORMULATION}

In classical radar estimation and detection problems, the signal parameter space (say $\Theta$ ) is often discretized into a grid $\mathcal{G}_{\Theta}$ (a discrete set), and all of as we don't know where an the signal/target parameter lies and how many are present. In practice, several values of the unkown parameter $\theta \in \mathcal{G}_{\Theta}$ are tested. Suppose we have an observation vector $\mathbf{y} \in \mathbb{C}^{P \times 1}$, which consists of a signal part corrupted by an additive Gaus- 
sian noise. The signal writes $\alpha \mathbf{s}(\theta) \in \mathbb{C}^{P \times 1}$, where $\alpha \in \mathbb{C}$ is the deterministic unkown signal amplitude and $\theta \in \Theta \subset \mathbb{R}$ is the parameter of interest. We assume that $\|\mathbf{s}(\theta)\|^{2}=1$. Let $\theta_{0} \in \mathcal{G}_{\Theta}$ be a given grid point (parameter under test) : the true target parameter $\theta$ can take any value in $\left[\theta_{0}-\frac{\Delta}{2}, \theta_{0}+\frac{\Delta}{2}\right]$, where $\Delta$ is the grid step. The grid step $\Delta$ is classicaly chosen so that $\mathbf{s}\left(\theta_{0}\right)$ and $\mathbf{s}\left(\theta_{0}+\Delta\right)$ are orthogonal. In the special case of interest, for the sinusoids-in-noise model

$$
\mathbf{s}(\theta)=\frac{1}{\sqrt{P}}\left[\begin{array}{llll}
1 & e^{j 2 \pi \theta} & \ldots & e^{j 2 \pi(P-1) \theta}
\end{array}\right]^{T},
$$

we have $\Delta=\frac{1}{P}$. This model is widely used, especially for radar target detection in Doppler domain. The noise is assumed complex circular Gaussian distributed $\mathbf{n} \sim \mathcal{C N}(\mathbf{0}, \mathbf{R})$, where $\mathbf{R}$ is unknown. Since $\mathbf{R}$ is unknown, it must be estimated via $K$ secondary i.i.d. data $\mathbf{y}_{k}=\mathbf{n}_{k}$ with $\mathbf{n}_{k} \sim$ $\mathcal{C N}(\mathbf{0}, \mathbf{R})$.

Then, the off-grid adaptive detection problem in Gaussian environment consists in deciding between two hypotheses: presence $\left(\mathcal{H}_{1}\right)$ or absence of a target $\left(\mathcal{H}_{0}\right)$ embedded in noise.

$$
\begin{gathered}
\mathcal{H}_{1}:\left\{\begin{array}{l}
\mathbf{y}=\alpha \mathbf{s}(\theta)+\mathbf{n}, \quad \theta \in\left[\theta_{0}-\frac{\Delta}{2}, \theta_{0}+\frac{\Delta}{2}\right], \\
\mathbf{y}_{k}=\mathbf{n}_{k}, \quad 1 \leq k \leq K,
\end{array}\right. \\
\mathcal{H}_{0}:\left\{\begin{array}{l}
\mathbf{y}=\mathbf{n}, \\
\mathbf{y}_{k}=\mathbf{n}_{k}, \quad 1 \leq k \leq K .
\end{array}\right.
\end{gathered}
$$

Since there is a mismatch between the parameter under test $\theta_{0}$ and the true target parameter $\theta$, classical detectors will endure a performance degradation. The criteria we study here is the mean detection probability over $\theta \in\left[\theta_{0}-\frac{\Delta}{2}, \theta_{0}+\frac{\Delta}{2}\right]$.

\section{ADAPTIVE SUBSPACE DETECTORS}

Since the signal $\mathbf{s}(\theta)$ belongs to a manifold, trying to approximate it by a vector $\mathbf{s}\left(\theta_{0}\right)$ (as it is implicitly done by classical detectors) does not lead to very robust tests. The idea here is to approximate the manifold by a proper subspace of dimension $p>1$. Then analytic performance of such tests under off-grid mismatch are derived.

\subsection{ASD and MAMF}

When we substitute the following subspace model

$$
\mathbf{y}=\mathbf{U} \boldsymbol{\alpha}+\mathbf{n}
$$

where $\boldsymbol{\alpha} \in \mathbb{C}^{p \times 1}$ and $\mathbf{U} \in \mathbb{C}^{P \times p}$ represents the signal subspace, to the model in (2), well known established detectors are [8] the Adaptive Subspace Detector (ASD),

$$
t_{A S D}=\frac{\| \mathbf{P}_{\mathbf{S}^{-\frac{1}{2}} \mathbf{U}^{\mathbf{z}} \|^{2}}}{K+\left\|\mathbf{P}_{\mathbf{S}^{-\frac{1}{2}} \mathbf{U}}^{\perp} \mathbf{z}\right\|^{2}} \underset{\mathcal{H}_{1}}{\stackrel{\mathcal{H}_{0}}{\lessgtr}} \eta_{A S D}
$$

which is the GLRT of the detection problem, the Multirank AMF (MAMF)

$$
t_{M A M F}=\frac{\left\|\mathbf{P}_{\mathbf{S}^{-\frac{1}{2}} \mathbf{U}} \mathbf{z}\right\|^{2}}{K} \underset{\mathcal{H}_{1}}{\stackrel{\mathcal{H}_{0}}{\lessgtr}} \eta_{M A M F},
$$

and the CFAR ASD (derived assuming that there is an unknown scale parameter in the noise variance between primary and secondary data),

$$
t_{C F A R-A S D}=\frac{\left\|\mathbf{P}_{\mathbf{S}^{-\frac{1}{2}} \mathbf{U}} \mathbf{z}\right\|^{2}}{\left\|\mathbf{P}_{\mathbf{S}^{-\frac{1}{2}} \mathbf{U}}^{\perp} \mathbf{z}\right\|^{2}} \underset{\mathcal{H}_{1}}{\stackrel{\mathcal{H}_{0}}{\lessgtr}} \eta_{C F A R-A S D},
$$

where $\mathbf{z}=\mathbf{S}^{-\frac{1}{2}} \mathbf{y}, \mathbf{S}=\frac{1}{K} \sum_{k=1}^{K} \mathbf{y}_{k} \mathbf{y}_{k}^{H}, \mathbf{P}_{\mathbf{U}}$ is the orthogonal projector on $\mathbf{U}$ and $\mathbf{P}_{\mathbf{U}}^{\perp}=\mathbf{I}-\mathbf{P}_{\mathbf{U}}$. Note that when $\mathbf{U}=\mathbf{s}\left(\theta_{0}\right)$, the ASD, the multirank AMF and the CFAR ASD are exactly Kelly's GLRT [5], the AMF [6] and the Adaptive Coherence Estimator (ACE), respectively.

\subsection{Performance of the ASD and MAMF under off-grid signal mismatch}

Following the lines of [8], it is possible to show that when the signal is modeled as (2), we can write the ASD, MAMF and CFAR ASD statistics as

$$
t_{A S D}=f, \quad t_{M A M F}=\frac{f}{b}, \quad t_{C F A R-A S D}=\frac{f}{1-b},
$$

where, conditionally to $b, f$ follows a non-central F-distribution: $f \mid b \sim F\left(\nu_{1}, \nu_{2}, b A(\theta)\right)$, with degrees of freedom $\nu_{1}=2 p$ and $\nu_{2}=2(K-P+1)$ and non-centrality parameter $A(\theta)=2|\alpha|^{2}\left\|\mathbf{P}_{\mathbf{R}^{-\frac{1}{2}} \mathbf{U}} \mathbf{R}^{-\frac{1}{2}} \mathbf{S}(\theta)\right\|^{2}$. Moreover, $b$ is such that $1-b$ follows a non-central Beta distribution: $1-b \sim$ $\beta\left(\nu_{3}, \nu_{4}, A^{\perp}(\theta)\right)$, with degrees of freedom $\nu_{3}=2(P-p)$ and $\nu_{4}=2(K-P+p+1)$ and non-centrality parameter $A^{\perp}(\theta)=2|\alpha|^{2}\left\|\mathbf{P}_{\mathbf{R}^{-\frac{1}{2}}}^{\perp} \mathbf{R}^{-\frac{1}{2}} \mathbf{S}(\theta)\right\|^{2}$. We can then deduce the probability of detection of the ASD, MAMF and CFAR ASD when the signal parameter equals $\theta$ :

$$
\begin{aligned}
P_{d}^{i}(\theta) & =\operatorname{Pr}\left[t_{i} \geq \eta_{i}\right] \\
& =1-\int_{0}^{1} F_{\left[\nu_{1}, \nu_{2}, b A(\theta)\right]}\left(\gamma_{i}(b)\right) p_{b, \theta}(b) d b
\end{aligned}
$$

where $i \in\{A S D, M A M F, C F A R-A S D\} . F_{\left[\nu_{1}, \nu_{2}, a\right]}(x)$ denotes here the cumulative distribution function of a noncentral F-distribution with degrees of freedom $\nu_{1}$ and $\nu_{2}$ and non-centrality parameter $a$. The distribution $p_{b, \theta}(b)$ of $b$ is the one explicited above. In order to obtain the distribution for either the ASD, the MAMF or the CFAR ASD, we only need to replace $\gamma_{i}(b)$ by either $\gamma_{A S D}(b), \gamma_{M A M F}(b)$, or $\gamma_{C F A R-A S D}(b)$ :

$$
\gamma_{A S D}(b)=\eta_{A S D} \frac{\nu_{1}}{\nu_{2}}, \quad \gamma_{M A M F}(b)=\eta_{M A M F} b \frac{\nu_{1}}{\nu_{2}},
$$




$$
\gamma_{C F A R-A S D}(b)=\eta_{C F A R-A S D}(1-b) \frac{\nu_{1}}{\nu_{2}} .
$$

The integration in (3) is performed numerically. Averaging with respect to $\theta \in\left[\theta_{0}-\frac{\Delta}{2}, \theta_{0}+\frac{\Delta}{2}\right]$ gives the desired mean detection probability $\bar{P}_{d}^{i}=\frac{1}{\Delta} \int_{\theta_{0}-\frac{\Delta}{2}}^{\theta_{0}+\frac{\Delta}{2}} P_{d}^{i}(\theta) d \theta$.

The false alarm probability $P_{f a}$ is simply obtained by setting $A(\theta)=A^{\perp}(\theta)=0$. The thresholds $\eta_{A S D}, \eta_{M A M F}$ and $\eta_{C F A R-A S D}$ are chosen in order to guarantee a desired $P_{f a}$.

Note that due to the mismatch, the result here differs from that in [8] where the distribution of $b$ was central Beta (versus non central Beta here) and the expression of the non centrality parameter in the F-distribution is also impacted.

\section{CHOICE OF THE SUBSPACE}

\subsection{Choosing the subspace $U$}

Assuming a fixed rank $p$, a good choice for the signal subspace is to choose an orthonormal basis $\mathbf{U}$ that minimizes the average projection error

$$
\mathbf{U}=\underset{\mathbf{V}, \operatorname{dim}(\mathbf{V})=p}{\arg \min } \mathcal{E}\left(\theta_{0}, \Delta, \mathbf{V}\right),
$$

where $\mathcal{E}\left(\theta_{0}, \Delta, \mathbf{V}\right)=\frac{1}{\Delta} \int_{\theta_{0}-\frac{\Delta}{2}}^{\theta_{0}+\frac{\Delta}{2}}\left\|\mathbf{s}(\theta)-\mathbf{V} \mathbf{V}^{H} \mathbf{s}(\theta)\right\|^{2} d \theta$. Such basis is then simply given by the $p$ eigenvectors (denoted $\mathbf{v}_{k}\left(\theta_{0}, \Delta\right)$ ) associated with the $p$ strongest eigenvalues of the matrix $\mathbf{U}\left(\theta_{0}, \Delta\right)=\frac{1}{\Delta} \int_{\theta_{0}-\frac{\Delta}{2}}^{\theta_{0}+\frac{\Delta}{2}} \mathbf{s}(\theta) \mathbf{s}^{H}(\theta) d \theta$ [9].

Moreover, the minimal projection residue is given by the sum of the remaining eigenvalues of $\mathbf{U}\left(\theta_{0}, \Delta\right)$.

$$
\min _{\mathbf{V}, \operatorname{dim}(\mathbf{V})=p} \mathcal{E}\left(\theta_{0}, \Delta, \mathbf{U}\right)=\sum_{k=p+1}^{P} \lambda_{k}\left(\theta_{0}, \Delta\right) .
$$

When the signal model is (1), we have

$$
\mathbf{U}\left(\theta_{0}, \Delta\right)=\frac{1}{\Delta}\left[\mathbf{s}\left(\theta_{0}\right) \mathbf{s}^{H}\left(\theta_{0}\right)\right] \odot \mathbf{B}_{P, \frac{\Delta}{2}},
$$

where $\odot$ denotes the Hadamard (element-wise) product, and the matrix $\left[\mathbf{B}_{P, W}\right]_{k l}=2 W \operatorname{sinc}(2 W(k-l)), 1 \leq k, l \leq P$, is the DPSS matrix [7]. Let us denote $\mathbf{u}_{k}(P, W)$ and $\lambda_{k}(P, W)$ the corresponding $\mathrm{k}$-th eigenvector and eigenvalue respectively (sorted by decreasing magnitude). Note that $\mathbf{v}_{k}\left(\theta_{0}, \Delta\right)=\mathbf{s}\left(\theta_{0}\right) \odot \mathbf{u}_{k}\left(P, \frac{1}{2 \Delta}\right)$. The index-limited sequence DPSS $\left\{\mathbf{u}_{n}(P, W)\right\}$ is by construction the indexlimited sequence with most concentrated spectrum in $[-W, W]$ and orthogonal to $\left\{\mathbf{u}_{k}(P, W), k \in[1, n-1]\right\}, \lambda_{k}(P, W)$ denoting its fraction of energy in $[-W, W][7]$.

\subsection{Choosing the dimension of the subspace $p$}

Choosing the optimal dimension $p$ of the subspace is not trivial. Increasing $p$ tends to reduce the projection error (the mismatch between the true and assumed signal subspace) and

\begin{tabular}{|c||c||c||c||c|}
\hline & $\rho=0$ & $\rho=0.3$ & $\rho=0.6$ & $\rho=0.9$ \\
\hline \hline $\operatorname{Pr}\left(\mathbf{n} \in \mathscr{C}_{\theta_{c}}\right)$ & $6.9 \times 10^{-4}$ & $1.4 \times 10^{-2}$ & 0.13 & 0.68 \\
\hline
\end{tabular}

Table 1: Probability for $\mathbf{n} \sim \mathcal{C N}(\mathbf{0}, \mathbf{R})$ to belong to the cone $\mathscr{C}_{\theta_{c}}$ centered in $\mathbf{s}\left(\theta_{0}\right)$ and of half angle $\Delta / 2 . \theta_{0}=0, \theta_{c}=$ $\cos ^{-1}\left(\frac{2}{\pi}\right)$. $\mathbf{R}$ is a symmetric Toeplitz matrix with coefficients $\left(1, \rho, \ldots, \rho^{P-1}\right), P=15$.

thus to increase the probability of detection. But increasing $p$ also tends to increase the threshold and thus, in turn, to decrease the detection probability. As in [4], we observed empirically that $p=2$ gives usually the best result. We have the following result [7]:

Theorem 1 When $W \rightarrow 0, P \rightarrow+\infty$ with $\pi W P \rightarrow c$, then $\lambda_{k}(P, W) \rightarrow \lambda_{k}(c)$, where $\lambda_{k}(c)$ is the $k-$ th eigenvalue associated with the $k-$ th Prolate Spheroidal Wave Function (PSWF) with corresponding parameter c [7].

In our case $W=1 / 2 P$ and $\pi W P=c=\pi / 2$. Thus when $P$ is large enough, the eigenvalue $\lambda_{k}(P, W)$ can be approximated by $\lambda_{k}(c)$. We have $\lambda_{1}(c) \approx 0.78, \lambda_{2}(c) \approx 0.20$, $\lambda_{3}(c) \approx 0.01$ and $\lambda_{4}(c) \approx 2.10^{-4}$. Thus, $p=2$ allows to capture around $98 \%$ of the signal subspace energy, considering the projection error (4).

\section{NUMERICAL RESULTS}

We consider here the case of steering vectors following the signal model (1). The SNR is here defined as $S N R=$ $20 \log _{10}(|\alpha|)$.

We assume covariance $\mathbf{R}$ is a symmetric Toeplitz matrix with coefficients $\left(1, \rho, \ldots, \rho^{P-1}\right)$ with $0 \leq \rho \leq 1$.

In order to implement the GLRT [3], we choose as signal subspace $\mathbf{s}\left(\theta_{0}\right)$ and a cone angle $\theta_{c}$ equivalent to a resolution cell half width, that is to say the angle between $\mathbf{s}\left(\theta_{0}\right)$ and $\mathbf{s}\left(\theta_{0}+\Delta / 2\right): \cos \left(\theta_{c}\right)=\frac{\left|\mathbf{s}\left(\theta_{0}+\Delta / 2\right)^{H} \mathbf{s}\left(\theta_{0}\right)\right|}{\left\|\mathbf{s}\left(\theta_{0}\right)\right\|_{2}\left\|\mathbf{s}\left(\theta_{0}+\Delta / 2\right)\right\|_{2}}=$ $\left|\frac{\sin (\pi / 2)}{P \sin (\pi / 2 P)}\right| \approx \frac{2}{\pi}$. Note that this angle is almost constant with respect to $P$ and is quite large (around $50^{\circ}$ ). As a consequence, the probability of $\mathbf{n} \sim \mathcal{C N}(\mathbf{0}, \mathbf{R})$ to belong to the cone - that sets a lower bound on the $P_{f a}$ - has to be computed. This probability for some scenario is studied in Table 1. As we can see when the application requires low $P_{f a}$ and/or when the noise exhibits some correlation, this technique is not suited anymore. Note that the bounded angle mismatch considered in [3] is more general than the off-grid mismatch, and since the mismatch cone contains more than the studied mismatch, it explains why it might not be very suited to our case.

In Figure 1, we compare the performances of the GLRT [3] and the proposed ASD, MAMF and CFAR ASD tests, called DPSS ASD, DPSS MAMF and DPSS CFAR ASD as we used DPSS subspace with $p=2$ for all of them. The 


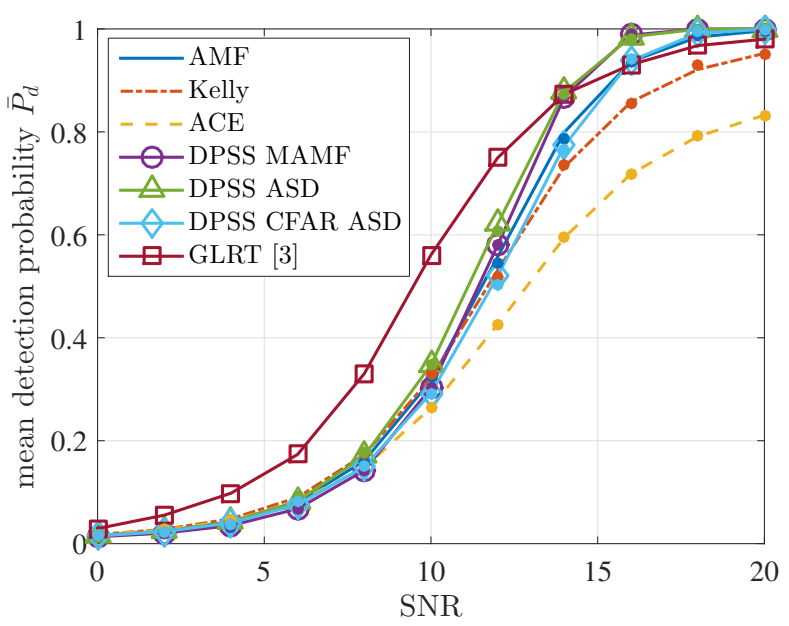

(a) $\rho=0, P_{f a}=5 \times 10^{-3}$

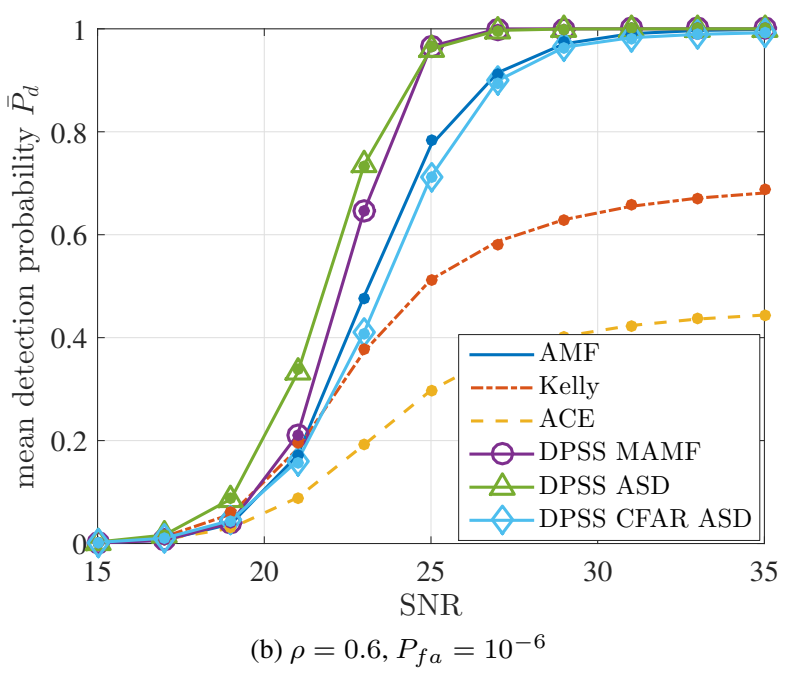

Fig. 1: Mean probability of detection $\bar{P}_{d}$ (obtained with derived theoretical expression). $P=15, \theta_{0}=0, \Delta=1 / P$, $K=2 P$. Dots represent Monte carlo runs (200 trials).

mean probability is computed by averaging 20 equi-spaced hypotheses within the resolution cell. As we can see in Figure 1a when the scenario is not too severe, the GLRT with bounded mismatch angle performs very well at low SNR. At higher SNR, the proposed detectors performs better. But when a low $P_{f a}$ is required, and when the noise exhibit some correlation (which is more a radar oriented context), the GLRT [3] can not be applied, since the $P_{f a}$ cannot be guaranteed. As can be seen in Figure 1b, Kelly's GLRT (known to be less robust to mismatch angle) suffer from severe performance loss, whereas the proposed subspace approaches perform quite well, outperforming the AMF. As expected, the CFAR ASD performs a little bit less than ASD and MAMF, since it assumes an additional unknown scaling parameter between primary and secondary data.

Other subspaces were tested, like subspaces build with equi-spaced replica of $\mathbf{s}(\theta)$ within the interval $\left[\theta_{0}-\frac{\Delta}{2}, \theta_{0}+\frac{\Delta}{2}\right]$ and showed lower performance than the proposed subspace.

\section{CONCLUSION}

In this paper we propose to use Adaptive subspace detectors in order to deal with the problem of off-grid target detection. Analytic performance of the detectors are provided as well as a suitable subspace. Interestingly, the proposed subspace is equal to the DPSS basis for the well-known sinusoids in noise. The proposed detectors showed good results on simulations, confirming their interest compared to the state of the art, especially for radar scenarii (with correlated noise, low probability of false alarm).

\section{REFERENCES}

[1] G. Tang, B. N. Bhaskar, P. Shah, and B. Recht, "Compressed sensing off the grid," IEEE transactions on information theory, vol. 59, no. 11, pp. 7465-7490, 2013.

[2] A. De Maio, "Robust adaptive radar detection in the presence of steering vector mismatches," IEEE Transactions on Aerospace and Electronic Systems, vol. 41, no. 4, pp. 1322-1337, 2005.

[3] O. Besson, "Detection of a signal in linear subspace with bounded mismatch," IEEE Transactions on Aerospace and Electronic Systems, vol. 42, no. 3, pp. 1131-1139, 2006.

[4] O. Rabaste, J. Bosse, and J.-P. Ovarlez, "Off-grid target detection with normalized matched subspace filter," 2016 24th European Signal Processing Conference (EUSIPCO), pp. 1926-1930, 2016.

[5] E. Kelly, "An adaptive detection algorithm," IEEE Transactions on Aerospace and Electronic Systems, vol. 2, no. AES-22, pp. 115-127, 1986.

[6] F. Robey, D. Fuhrmann, E. Kelly, and R. Nitzberg, "A cfar adaptive matched filter detector," IEEE Transactions on Aerospace and Electronic Systems, vol. 28, no. 1, pp. 208-216, 1992.

[7] D. Slepian, "Prolate spheroidal wave functions, fourier analysis, and uncertainty-V: The discrete case," Bell System Technical Journal, vol. 57, no. 5, pp. 1371-1430, 1978.

[8] S. Kraut, L. L. Scharf, and L. T. McWhorter, "Adaptive subspace detectors," IEEE Transactions on signal processing, vol. 49, no. 1, pp. 1-16, 2001.

[9] J. Bosse and O. Rabaste, "Subspace rejection for matching pursuit in the presence of unresolved targets," IEEE Transactions on Signal Processing, vol. 66, no. 8, pp. 1997-2010, 2018. 